\title{
SIMULATIONS OF AUTOIGNITION AND LAMINAR PREMIXED FLAMES OF METHANE-AIR MIXTURES DILUTED WITH HOT PRODUCTS
}

\author{
J. Sidey ${ }^{1} *$, E. Mastorakos ${ }^{1}$, and R. L. Gordon ${ }^{2}$ \\ 1: Department of Engineering, University of Cambridge, Cambridge CB2 1PZ, UK \\ 2: Rolls Royce Canada Ltee, Montreal, Canada \\ * Corresponding author: jams4@cam.ac.uk
}

\begin{abstract}
This paper considers constant-pressure autoignition and freely propagating premixed flames of cold methane-air mixtures mixed with equilibrium hot products at high enough dilution levels that the combustion enters the MILD combustion regime. The analysis is meant to provide further insight on MILD regime boundaries and to identify the effect of hot products speciation. As the mass fraction of hot products in the reactants mixture increases, autoignition occurs earlier. Species profiles show that the products-reactants mixture approximately equilibrates to a new state over a quick transient well before the main autoignition event, but as dilution becomes very high, this equilibration transient becomes more prominent and eventually merges with the primary ignition event. The dilution level at which these two reactive zones merge corresponds well with that marking the transition into the MILD regime, as defined according to conventional criteria. Similarly, premixed flame simulations at high dilutions show evidence of significant reactions involving intermediate species prior to the flame front. Since the premixed flame governing equations system demands that the species and temperature gradients be zero at the "cold" boundary, flame speed cannot be calculated above a certain dilution level. Up to this point, which again agrees reasonably well with the transition into the MILD regime according to convention, the laminar burning velocity was found to increase with hot product dilution while flame thickness remained largely unchanged. Some comments on the MILD combustion regime boundary definition for gas turbine applications are included.
\end{abstract}

\section{Introduction}

The drive for low-emission combustion technologies has sparked extensive interest in Moderate to Intense Low Oxygen Dilution, or MILD, combustion [1]. MILD combustion involves preheating and diluting fresh reactants and is defined as the condition where the resulting mixture temperature is higher than its autoignition temperature, while the total temperature rise during combustion is lower than this autoignition temperature [1]. Note that, despite the fact that the concept of "autoignition temperature" is not a fundamental characteristic of a combustion system, this definition can help delineate the MILD regime boundary for operational purposes; this will be discussed later in this paper. Dilution and preheating may be performed simultaneously by mixing reactants with hot combustion products, often in a closed combustor or furnace environment, which implies that the degree of preheating and the degree of dilution are intimately connected. One of the key characteristics by which MILD combustion is achieved in practice is to inject the air and the fuel from small enough fast jets into a highly recirculating flow of hot products so that mixing can occur very quickly relative to the residence time in the furnace. This suggests that the special case of adiabatic mixing of hot equilibrium products with the cold reactants, and the subsequent combustion behaviour of the diluted mixture, is particularly relevant. 
Multiple numerical studies have addressed facets of MILD combustion, and here we review only previous work related to examination of canonical flame structures such as homogenenous reactor autoignition and premixed and non-premixed laminar flames. De Joannon et al. [2] investigated the dependence of autoignition delay time on oxygen concentration and temperature of premixed heavy hydrocarbons near 4 bar. They reported a negative power law dependence of autoignition delay time on oxygen mole fraction and classified regimes of MILD ignition behavior based on inlet temperature while identifying the primary reaction pathways within these regimes. De Joannon et al. [3,4,5] continued their work of identifying steady combustion sub-regimes associated with MILD conditions with their investigation of diluted non-premixed counterflow flames. Through their regime identification studies, they observed that high temperature dilution results in counterflow flames with broad heat release regions and uniformly distributed temperature profiles in mixture fraction space; a finding which supports the empirical description of MILD combustion as a dispersed or distributed reaction. They also reported that transition regions surrounding their identified MILD regimes were continuous and gradual, consistent with the reduced noise characteristics of highly preheated and MILD combustion.

More recently, numerical MILD combustion studies have again become focused on capturing and explaining the autoignition properties of non-premixed, diluted structures previously studied in a steady context. De Joannon et al. [6] continued investigating diluted counterflow diffusion flames and commented on MILD chemistry, concluding that reactant dilution with an inert species slows down oxidative chemical kinetics and increases the distance between the most reactive and stoichiometric mixture fractions. Recently, Abtahizadeh et al. [7] performed a similar study investigating the autoignition of diluted and preheated reactants under various fuel, oxidant, and mixture dilution conditions using hot products rather than an inert species as a dilutant. They found that, in agreement with Ref. [6], autoignition behavior depends on complex chemical kinetics which vary with dilution method. They also observed that ignition was promoted by hot product dilution not only due to preheat temperature, but likely also due to the triggering of chain branching reactions by $\mathrm{H}_{2}$ and $\mathrm{CH}_{2} \mathrm{O}$ mole fractions in the hot product dilutant [7]. These findings are well supported by further studies performed Sabia et al. [8,9] who studied the autoignition delay time of methane mixtures diluted with $\mathrm{N}_{2}, \mathrm{H}_{2} \mathrm{O}$, and $\mathrm{CO}_{2}$. They concluded that high preheat and dilution levels slowed down kinetic pathways during combustion, particularly by stressing the competition between oxidation and recombination channels, however, reaction speed was dependent on the nature of the dilutant. Sabia et al. reported slower autoignition times by diluting with inert species in than those reported in the hot product dilution study performed by Abtahizadeh et al., supporting the assumption that minor species present in dilutant mixtures promote ignition by initiating chain branching reactions $[7,8]$.

Despite the success of these investigations, a significant amount of work remains to be done in order to completely understand the fundamentals of MILD combustion and the effects of hot product dilution. In particular, the case of simultaneous dilution and preheating with hot products has not been studied thoroughly. Examination of the previously-cited papers, of the well-stirred reactor simulations of Ref. [10], and of extrapolation of laminar counterflow flame theoretical analysis to high dilution and preheat conditions [11], shows that one of the emerging characteristics of MILD combustion is the absence of critical points marking ignition and extinction. Indeed, in the experiments of Ref. [11], extinction of a non-premixed flame between fuel and a hot products/air mixture was never observed at high oxidiser temperatures, despite the corresponding high dilution, and similarly for premixed flames in the stagnation region of a cold premixed reactants jet against a hot products jet. The absence of a well-defined sudden extinction at high opposed jet temperatures is also seen in theoretical work [12]. Ignition and extinction are fundamental concepts related to the high activation 
temperature relative to the "cold" temperature of the reactants, and this is also the basis behind the concept of laminar burning velocity, which assumes a sharp transition between chemically-frozen reactants and equilibrium products. It is of fundamental value to examine if laminar burning velocity can be defined in the MILD combustion regime.

This work is aimed at providing insight into the structure of premixed flames and the autoignition of homogeneous mixtures as they become increasingly diluted with hot combustion products. The particular feature of the present simulations is that the dilution and the preheat are not independent, but are related by considering adiabatic mixing of the fresh reactants at a set equivalence ratio with the equilibrium hot products (i.e. at the adiabatic flame temperature) of this same equivalence ratio. This special case corresponds precisely to the mixing of the injected reactants with the recirculated products in a typical MILD combustor. Defining the unburnt state as a mixture of cold reactants and equilibrium hot products is an idealisation of the hypothesis that, in MILD combustion, mixing of hot products and the fresh reactants is assumed to occur infinitely fast. This paper also provides evidence that supports the conjecture that the MILD combustion boundary is the point where the laminar premixed flame ceases to exist in a conventional sense. A preliminary method for identifying working conditions for MILD combustion gas turbine operation is suggested.

\section{Method}

The most common ways to characterise the combustion behaviour of a homogeneous mixture are to examine its autoignition and the laminar flame that may propagate into such a mixture. Both these canonical problems are studied here numerically at constant, atmospheric pressure. The homogeneous mixtures are prepared in the following manner. Three unstrained premixed flames of methane-air mixtures at $298 \mathrm{~K}$ and at lean $(\varphi=0.6)$, stoichiometric and rich $(\varphi=$ 1.3) conditions at atmospheric pressure were first calculated with PREMIX [13] and the GRI 3.0 mechanism [14]. The domain length was large enough $(40 \mathrm{~mm})$ that at the exit the hot gases were virtually in chemical equilibrium at the adiabatic flame temperature. These are called "hot products" in this paper. (Note that a chemical equilibrium solver could have been used for defining this state, but the differences due to the finite length of the domain are immaterial for the purposes of this paper.) These hot products were then mixed at a set proportion by mass with the cold reactants of the same equivalence ratio, and the degree of mixing was given by the "dilution variable", $\zeta$. Undiluted cold reactants are defined as $\zeta=0$, while pure hot products at the adiabatic flame temperature correspond to $\zeta=1$. Enthalpy remains constant during this mixing process and the final mixture temperature was determined by the enthalpy and the species mass fractions. This mixing procedure was used to obtain reactant/product mixtures for lean $(\varphi=0.6)$, stoichiometric and rich $(\varphi=1.3)$ conditions and at increments in $\zeta$ of 0.1 .

In addition to these mixtures, hereafter referred to as "full" as they contain every species found in the associated undiluted flame's hot products, a numerical experiment was performed involving what will now be referred to as "reduced" mixtures. Rather than mixing fresh reactants with all the hot products from the premixed flame of the same equivalence ratio, fresh reactants in the reduced mixtures were combined with only the major species $\mathrm{O}_{2}$, $\mathrm{CH}_{4}, \mathrm{CO}_{2}, \mathrm{H}_{2} \mathrm{O}, \mathrm{CO}$, and $\mathrm{H}_{2}$. The mass fractions of the removed species were added to the nitrogen. These reduced product/reactant mixtures therefore only contain $\mathrm{CH}_{4}, \mathrm{O}_{2}, \mathrm{~N}_{2}, \mathrm{CO}_{2}$, $\mathrm{H}_{2} \mathrm{O}$, CO, and $\mathrm{H}_{2}$, while the full dilution mixtures contain all 52 species found in the GRI 3.0 mechanism. The reduced mixtures will be used later on in this analysis to isolate the effect of radical species on autoignition.

The simulation of autoignition at constant pressure was performed with the adiabatic plug flow reactor (PFR) module of CHEMKIN and the GRI 3.0 chemistry. The ignition delay 
time $\tau_{\text {ign }}$ is defined as the time at which the temperature is midway between the mixture preheat temperature and the final temperature reached. The simulation of a one-dimensional, laminar, adiabatic, steady, freely propagating premixed flame at atmospheric pressure was performed with CHEMKIN's PREMIX module and the same chemistry. Mixture-averaged transport properties were used. The laminar burning velocity, $S_{\mathrm{L}}$, is calculated as an eigenvalue of the system of conservation equations (see, for example, [15]). In PREMIX, the numerical method proceeds by fixing the location of flame as a temperature point in the domain, which should be long enough to ensure that the temperature and species gradients vanish at the cold and hot boundaries. If $L$ is the distance of the cold boundary from the flame, then $L / S_{\mathrm{L}}$ can be thought of as the "time of flight" of the reactants until they reach the flame. In the case of chemically-frozen reactants, the flame will be the same no matter what $L$ may be. In the case of very weak chemical reactions, the differences in the flame structure are expected to be small. However, in the case of chemically active reactants, as expected under MILD conditions, the "cold" boundary condition of zero gradient is not satisfied anymore and the eigenvalue $S_{\mathrm{L}}$ may cease to exist (in practice, manifested by PREMIX failing to provide a solution). It is clear therefore that the conventional manner of calculating laminar burning velocity will be seriously affected as the MILD regime is reached, and this is used here as a diagnostic for identifying approximately the MILD combustion boundary.

\section{Results and Discussion}

\section{Autoignition of homogeneous mixtures}

The temperature evolution of various mixtures (equivalence ratios, $\varphi=0.6,1.0$, and 1.3) and at dilution levels spanning the whole range 0 to 1 , are shown in Fig. 1. Lightly diluted mixture $(\zeta=0.2$ to 0.4$)$ ignition events are characterized by a sharp jump from the preheat temperature to the adiabatic flame temperature. At higher levels of dilution $(\zeta=0.5-0.7)$, this jump in temperature becomes more gradual, with small gradients both before and after the primary temperature increase. At very high dilution levels (above $\zeta=0.8$ ), the profiles have become smooth, gradual reactions from a high initial temperature to the adiabatic flame temperature. Particularly at rich and stoichiometric conditions, no clear autoignition event can be distinguished from what, at moderate dilutions, appeared to be minor reactions immediately before and after the event itself.

The ignition delay time of each diluted mixture is shown as a function of dilution level in the bottom right graph of Fig. 1 . The $\tau_{\text {ign }}$ of the full mixtures, referring to those mixed with all 52 product species, are marked with solid symbols, while the $\tau_{\text {ign }}$ of the reduced mixtures, referring to reactants mixed with only $\mathrm{O}_{2}, \mathrm{CH}_{4}, \mathrm{CO}_{2}, \mathrm{H}_{2} \mathrm{O}, \mathrm{CO}$, and $\mathrm{H}_{2}$, are marked with open symbols. As both mixtures become more diluted, they ignite earlier with the exception of the stoichiometric case whose ignition delay time is minimized at $\zeta=0.7$. The rich and stoichiometric mixtures ignite earlier than the lean, most probably due to their higher preheat temperatures. However, regardless of equivalence ratio and dilution level, full mixtures consistently ignite earlier than the reduced mixtures. This suggests that ignition is encouraged by the presence of the minor species in the combustion products, a finding consistent with those of Abtahiadeh et al. [7]. Some of these species, such as $\mathrm{H}$ and $\mathrm{OH}$, are important participants in chain branching reactions which initiate the combustion process. Further still, hot product species would affect methane oxidation channels by increasing the efficiency of some important reactions as a third body nonparticipant. It is possible that some species promote chain branching reactions this way, thus encouraging ignition. 
Intermediate species profiles, specifically $\mathrm{CH}_{2} \mathrm{O}, \mathrm{CH}_{3}, \mathrm{OH}$, and $\mathrm{H}_{2}$, for each equivalence ratio and dilution level are shown in Fig. 2. The lean, $\varphi=0.6$ profiles show a short, sharp spike in all radicals, corresponding to each mixture's ignition event. At higher dilution levels, the increase in intermediate mole fraction associated with the ignition event becomes longer and more drawn out, indicating that ignition occurs over a longer period of time. This result is consistent with those reported by Sabia et al. [8] who suggested that dilution significantly slows kinetic pathways.

The $\varphi=0.6 \mathrm{CH}_{3}$ profiles show that, at high dilutions (say, above $\zeta=0.4$ ) an initial reacting region, about $0.1 \mathrm{~ms}$ long, occurs immediately upon starting the simulation. This transient allows the fresh reactants-hot products mixture to reach a quasi-equilibrium state, as the species present in the hot products adapt to the colder temperature. Some exothermic reactions may occur in this period, in turn causing small increases in temperature before ignition in moderate to intensely diluted mixtures. As dilution increases, the time until the main ignition decreases, and this initial equilibration reaction merges with the mixture's
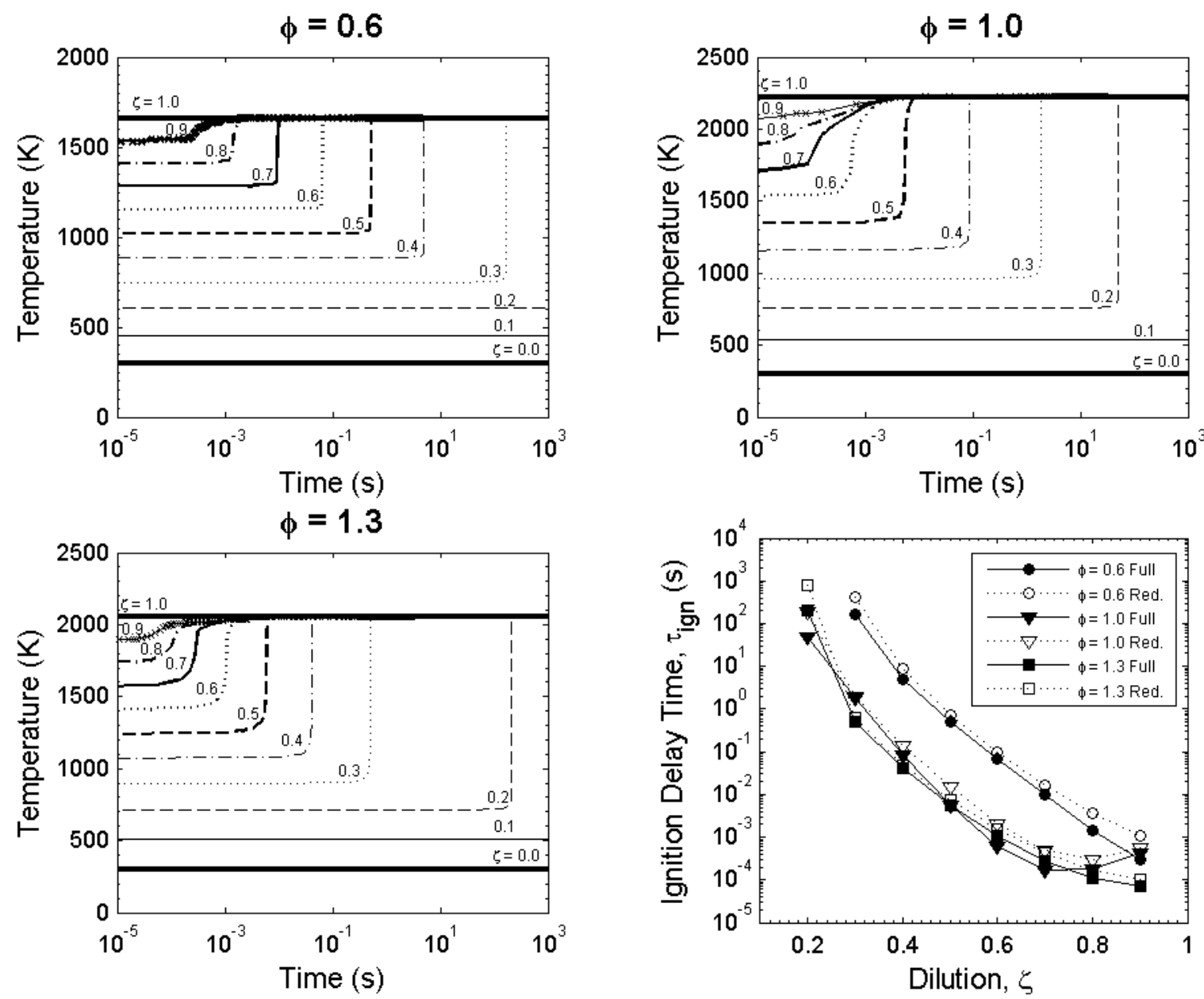

Figure 1. Temperature evolution of mixtures diluted with hot products at different dilution levels (marked on the curve) in a plug flow reactor. The bottom right image shows ignition

delay time varying with dilution level at different equivalence ratios for both the full (corresponding to the preceding temperature profiles) and reduced (abbreviated as "Red.") product mixtures. 

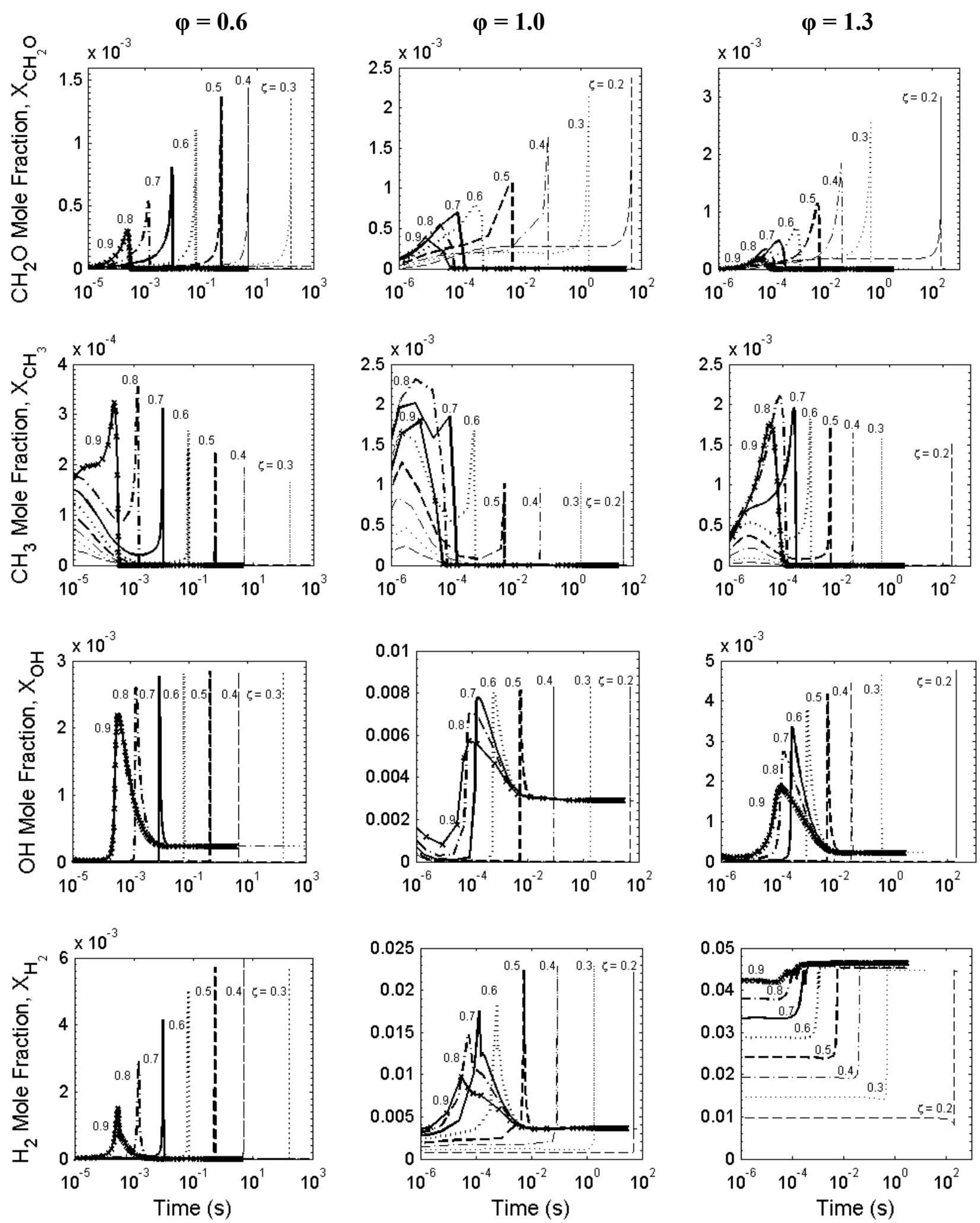

Figure 2. $\mathrm{CH}_{2} \mathrm{O}, \mathrm{CH}_{3}, \mathrm{OH}$, and $\mathrm{H}_{2}$ species profiles during the autoignition of full diluted mixtures. Lean, $\varphi=0.6$ profiles are shown on the left, stoichiometric, $\varphi=1.0$, profiles are shown in the center, and rich, $\varphi=1.3$, profiles are shown on the right. 
primary ignition event. This can be seen clearly in the $\varphi=0.6, \zeta=0.9, \mathrm{CH}_{3}$ species profile, where the pre-ignition and ignition reactions have combined to form one elongated event. The merging of these two otherwise distinct reactions results in a temperature profile lacking an obvious and sharp ignition event, displaying only a gradual rise in temperature occurring over the combined pre-ignition and ignition duration.

These trends can also be seen in the $\varphi=1.0$ and 1.3 cases. Species such as $\mathrm{CH}_{2} \mathrm{O}$ and $\mathrm{OH}$ show a wider and shorter peak at higher dilutions. Similarly, the $\mathrm{H}_{2}$ profile shows significant changes in mole fraction over the duration of the ignition event for all dilution cases. At high dilutions, the $\varphi=1.0 \mathrm{H}_{2}$ profiles show a wide and short peak before reaching a post ignition equilibrium value, while the $\varphi=1.3$ profiles mirror their associated temperature profiles as the post-reaction $\mathrm{H}_{2}$ mole fraction is very high. As in the lean case, at high dilutions, the pre-ignition equilibrium reaction merges with the primary ignition event, as shown by the stoichiometric and rich $\mathrm{CH}_{3}$ profiles.

\section{Premixed flames}

The temperature profiles for the three equivalence ratios studied and for the dilutions up to which a solution can be found are shown in Fig. 3, along with $\mathrm{CH}_{2} \mathrm{O}$ species profiles and results for the burning velocity and the flame thickness. The lowest curve on each graph corresponds to the temperature profile of the least diluted mixture, i.e. the mixture with the highest fraction of fresh reactants and thus the lowest preheat temperature. At all equivalence ratios, most mixtures, particularly those with low levels of dilution, produce a premixed flame with a usual temperature profile showing a stable preheat zone, a sudden increase in temperature corresponding to a flame front, and a post reaction zone at which burned gases eventually reach the adiabatic flame temperature. However, once the mixtures become highly diluted, the code fails to give a flame-like solution. In the lean case $(\varphi=0.6)$, the last such solution achieved was at $\zeta=0.6$ and $T_{i}=1335 \mathrm{~K}$. In the stoichiometric case, the code fails above a lower dilution level, $\zeta=0.48$, where the preheat temperature is $T_{i}=1282 \mathrm{~K}$. At $\varphi=$ 1.3 , a flame-like solution can be obtained up to $\zeta=0.6$ and $T_{i}=1572 \mathrm{~K}$. Some of the flames at high dilution show a significant temperature increase early in the preheat zone, far upstream from the flame. The rich $\zeta=0.6$ temperature profile also shows a slow and gradual temperature rise in the post reaction zone. Further still, the flame front itself is very wide compared to the lower diluted cases.

For a narrow range of dilution levels and just before the code's catastrophic behaviour, the code gives a solution as indicated in Fig. 3 with the $\zeta=0.7$ case for $\varphi=0.6$, the $\zeta=0.5$ case for $\varphi=1.0$, and the $\zeta=0.7$ case for $\varphi=1.3$. The corresponding eigenvalues given by the code are $21.3,79.5$, and $47.6 \mathrm{~cm} / \mathrm{s}$ respectively, which are lower than the last flame-like burning velocities (Fig. 3, lower). These structures can be thought of as stable solutions of the system of equations and the chosen domain, where the reaction is located almost right at the entry, which implies heat loss and a reduction in $S_{\mathrm{L}}$. Above these dilutions, the code fails to give any solution at all. We can identify therefore the emergence of solutions such as those discussed here as the point where the behaviour of the system ceases to be flame-like.

The burning velocity and flame thickness are displayed in Fig. 3. Flame speed increases with increasing dilution, an expected result considering the higher reactants with hot product mixing. (The values of the pathological solutions described above are not included.) Finally, the laminar flame thickness $\delta_{\mathrm{L}}$, obtained by finding the distance between the intersection points of the tangent line at the maximum slope of the flame temperature profile with the initial preheat and final temperatures, remains essentially unchanged as dilution increases for each equivalence ratio, once more with the exception of the $\varphi=1.3 \zeta=0.6$ case. 

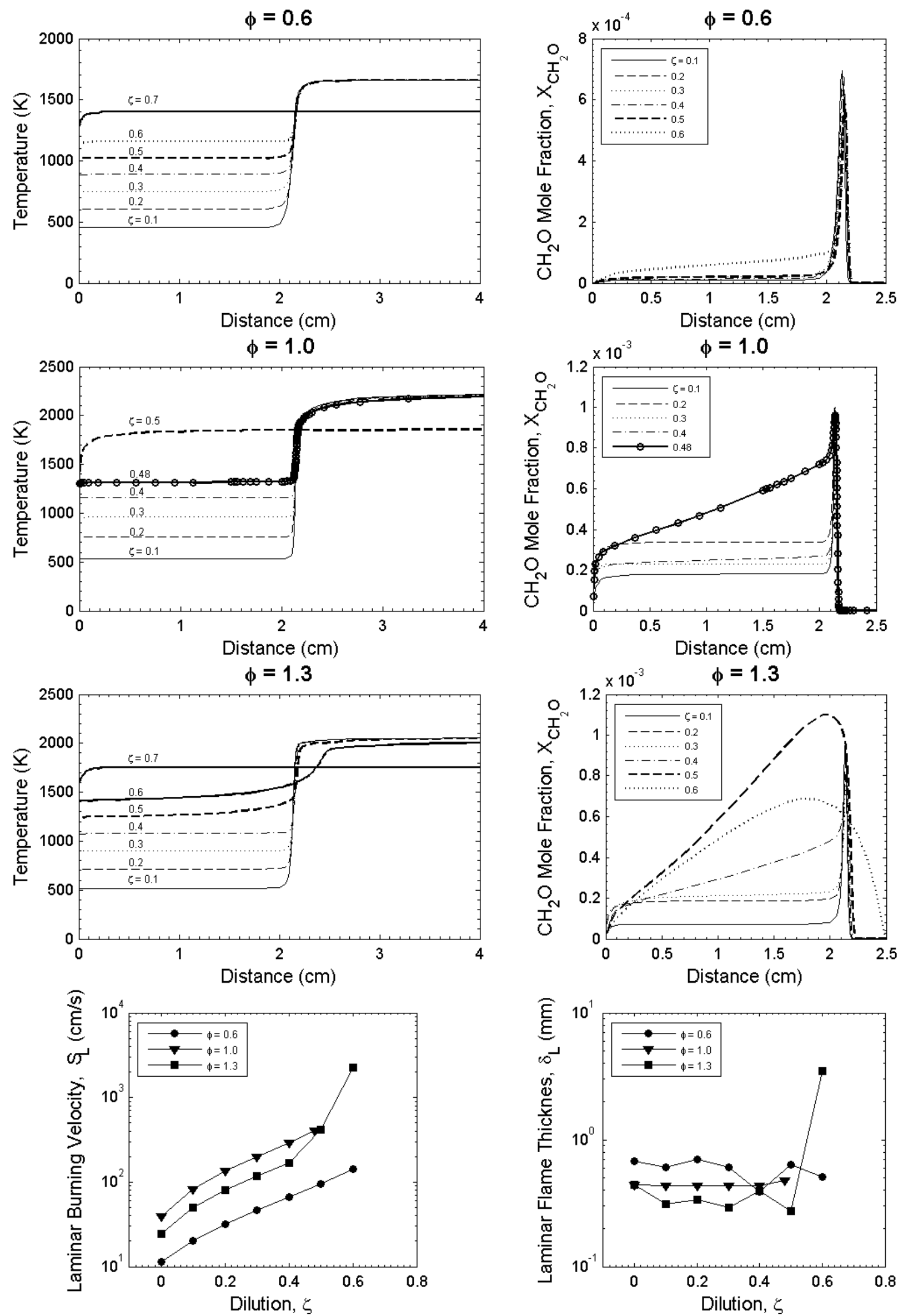

Figure 3. Temperature (left) and $\mathrm{CH}_{2} \mathrm{O}$ (right) profiles of diluted premixed flames at equivalence ratios of $\varphi=0.6,1.0$, and 1.3. Additionally, laminar burning velocity, $\mathrm{S}_{\mathrm{L}}$, (bottom left) and flame thickness, $\delta_{\mathrm{L}}$, (bottom right) are shown varying with dilution, $\zeta$, for $\varphi=0.6,1.0$, and 1.3 

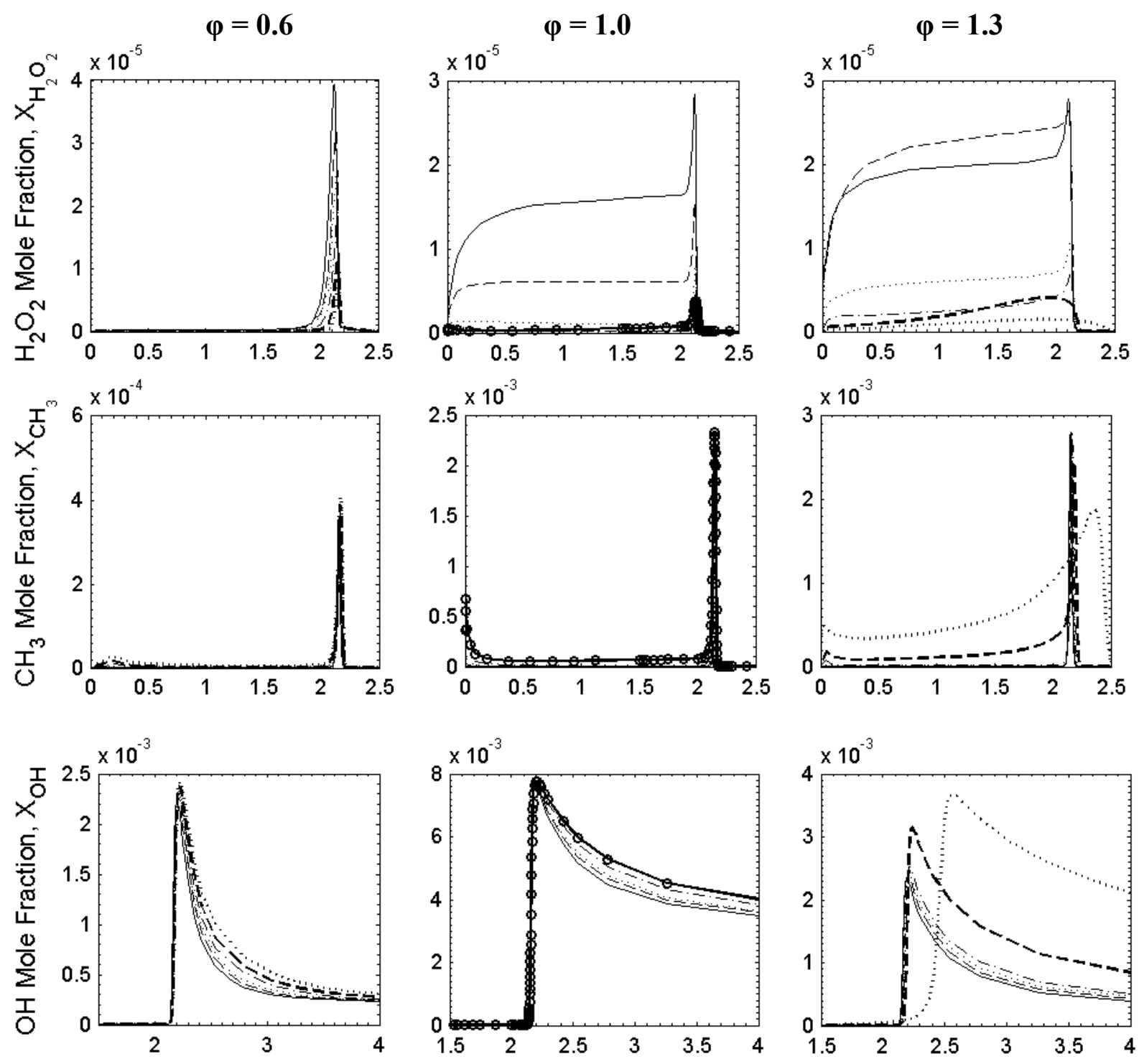

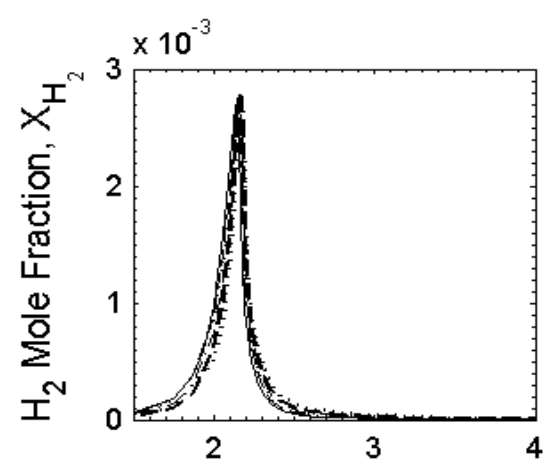

Distance $(\mathrm{cm})$

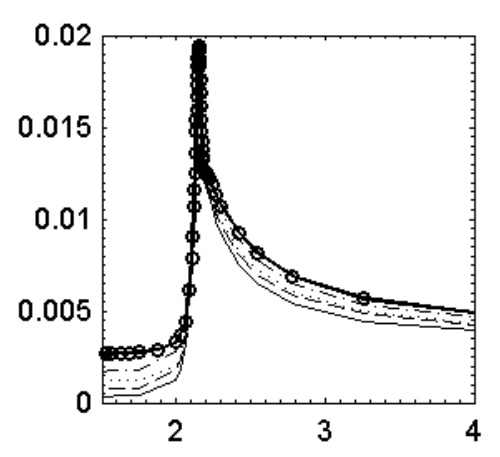

Distance $(\mathrm{cm})$

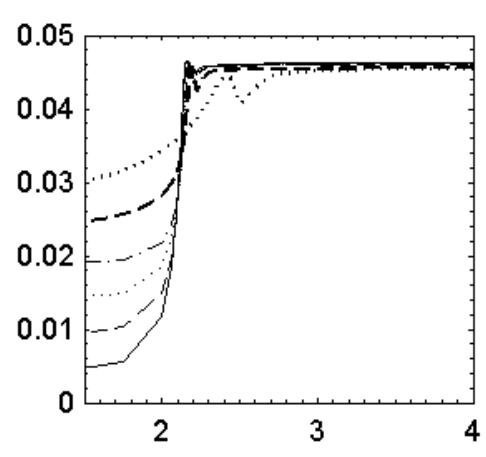

Distance $(\mathrm{cm})$

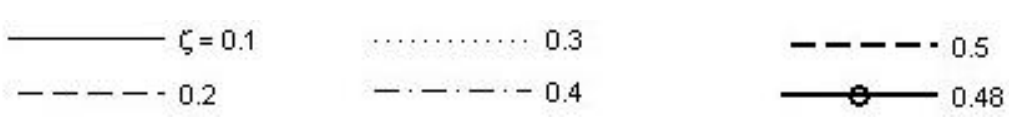

Figure 4. $\mathrm{H}_{2} \mathrm{O}_{2}, \mathrm{CH}_{3}, \mathrm{OH}$, and $\mathrm{H}_{2}$ species profiles of premixed diluted flames. Lean, $\varphi=0.6$ profiles are shown on the left, stoichiometric, $\varphi=1.0$, profiles are shown in the center, and rich, $\varphi=1.3$, profiles are shown on the right. 
Using $\delta_{\mathrm{L}}=v / S_{\mathrm{L}}$, where $v$ is the kinematic viscosity, and since $v$ is expected to increase with dilution and we have seen (Fig. 3) that $S_{\mathrm{L}}$ also increases with dilution, the approximately constant thickness of the flame can be understood. This trend is also consistent with the finding of Ref. [16] who demonstrated thin reaction zones in DNS of turbulent premixed combustion in the MILD combustion regime.

The $\mathrm{CH}_{2} \mathrm{O}$ intermediate profiles are shown in Fig. 3, while $\mathrm{H}_{2} \mathrm{O}_{2}, \mathrm{CH}_{3}, \mathrm{OH}$ and $\mathrm{H}_{2}$ profiles are displayed in Fig. 4. In the stoichiometric and, primarily, rich case, species which typically appear before ignition such as $\mathrm{CH}_{2} \mathrm{O}, \mathrm{H}_{2} \mathrm{O}_{2}$, and $\mathrm{CH}_{3}$ are produced in significant quantities before the flame front. This further supports the conclusion that as dilution increases significantly, the premixed flame approaches an autoignition problem. High temperature intermediates, like $\mathrm{OH}$ and $\mathrm{H}_{2}$, show evidence of increased activity after the flame front for high dilution cases. Finally, in agreement with the temperature profiles in Fig. 3 , the $\varphi=1.3 \zeta=0.5$ and $\zeta=0.6$ cases react very gradually in wide flame fronts. These rich, high dilution cases show the simultaneous build-up of autoignition radicals as well as localized high temperature radicals within a wide flame front, further suggesting that the premixed flame approaches an autoignition problem as dilution increases.

\section{MILD combustion in gas turbines}

Cavaliere and de Joannon [1] define MILD combustion as any combustion process which involves reactants preheated above their mixture's autoignition temperature where the temperature rise during combustion is lower than the autoignition temperature. The term "autoignition temperature" can be defined in various ways, but if we use here the conventional definition that it is the lowest temperature below which any fuel-air mixture at a given pressure will not ignite in a standard testing vessel, then for methane and atmospheric pressure this temperature is quoted as $873 \mathrm{~K}$ [17]. Similarly defined autoignition temperatures have also been measured for higher pressures [18] and so, for the benefit of the discussion that follows, assume that the autoignition temperature of the fuel has been measured in standard testing procedures for pressure $P$. Assume the combustor is considered as a plugflow reactor with incoming mixture at equivalence ratio $\varphi$, inlet temperature $T_{\mathrm{in}}$, and constant pressure $P$. If the flow rate is $\dot{m}$ and the volume of the combustor $V$, then the residence time $\tau_{\text {res }}$ is given by $\tau_{\text {res }}=\dot{m} / \rho V$, where $\rho$ is the density in the combustor (determined by the pressure and the adiabatic flame temperature corresponding to $\varphi, P$, and $T_{\text {in }}$ ). Then, we can postulate that MILD combustion with internal hot product recirculation may occur in the combustor if: (i) $\tau_{\text {res }}>\tau_{\text {ign }}$, where $\tau_{\text {ign }}$ is the ignition delay time as calculated in this paper (i.e. with the preheat and dilution related by adiabatic mixing of hot products and fresh reactants); and (ii) the conditions of Cavaliere and de Joannon [1] are satisfied. The combination of these two prerequisites will allow the dilution level $\zeta$ to be selected given the operating conditions $\left(\varphi, P, T_{\text {in }}\right.$, and $\left.\dot{m}\right)$ and geometry $(V)$. For example, a combustor with ambient inlet conditions (298K and 1 bar) with a residence time of $1 \mathrm{~ms}$ necessitates $\zeta$ greater than $0.8,0.6$, and 0.6 for $\varphi=0.6,1.0$, and 1.3 respectively to satisfy condition (i) (from Fig. 3), which happen to be high enough values of $\zeta$ that the initial temperature (i.e. after mixing with the hot products) is high enough for conditions (ii) to be automatically satisfied.

Note that the value of $\zeta$ at which the laminar premixed flame ceases to exist in the simulations is not far from the value of $\zeta$ one would select by looking only at the Cavaliere and de Joannon [1] conditions, but the extra condition that the ignition must occur inside the combustor imposes a more refined regime boundary that takes into account the finite ignition delay times even of very highly preheated mixtures. Note that performing similar calculations for mixtures with preheating but without the accompanying dilution, which are necessarily and inextricably linked in an adiabatic mixing situation, could give ignition delay times in grave error. 


\section{Conclusions}

A numerical study involving methane mixtures diluted with hot combustion products autoigniting in a plug flow reactor and burning as a premixed flame has been performed. With extensive hot product dilution, autoignition events begin to lose their characteristic sharply increasing temperature rise. Numerical experiments showed that the presence of minor species in the hot product definition can affect the ignition delay time. For high levels of hot product mixing, the initial quick equilibration zone eventually merges with the primary ignition event. The merging of these two zones can be considered a characteristic of MILD combustion. Premixed flame burning velocities could not be obtained for high dilution levels because temperature and species gradients were not negligible at the cold boundary, invalidating therefore the conditions necessary for a uniquely-defined laminar burning velocity to exist. Intermediate species profiles show evidence of significant chemical reaction far upstream from the flame front, implying that as the MILD regime is encountered, the premixed flame becomes akin to an autoignition problem. The point at which the premixed flame code fails to provide a solution corresponds well with the transition into the MILD combustion regime as defined conventionally. The use of the present calculation strategies for identifying the MILD combustion regime boundaries for gas turbines is discussed.

\section{Acknowledgments}

This work has been partly funded by Rolls-Royce plc. We wish to thank Prof. A. Cavaliere and Drs. M. de Joannon and P. Sabia of University of Naples for useful discussions on MILD combustion.

\section{References}

[1] Cavaliere, A., de Joannon, M., "Mild combustion", Prog. Energy Combust. Sci., 30: 329-366 (2004)

[2] de Joannon, M., Cavaliere, A., Donnarumma, R., Ragucci, R., "Dependence of autoignition delay on oxygen concentration in mild combustion of high molecular weight paraffin", Proc. Combust. Inst., 29: 1139-1146 (2002)

[3] de Joannon, M., Sabia, P., Sorrentino, G., Cavaliere, A., "Numerical study of mild combustion in hot diluted diffusion ignition (HDDI) regime", Proc. Combust. Inst., 32: 3147-3154 (2009)

[4] de Joannon, M., Sorrentino, G., Cavaliere, A., "MILD combustion in diffusioncontrolled regimes of Hot Diluted Fuel", Combust. Flame, 159: 1832-1839 (2012)

[5] de Joannon, M., Sabio, P., Cozzolino, G., Sorrentino, G., Cavaliere, A., "Pyrolitic and oxidative structures in hot oxidant diluted oxidant (HODO) MILD combustion", Combust. Sci. and Tech., 184: 1207-1218 (2012)

[6] Sorrentino, G., Scarpa, D., Cavaliere, A., "Transient inception of MILD combustion in hot diluted diffusion ignition (HDDI) regime: A numerical study", Proc. Combust. Inst., 34: 3239-3247 (2013)

[7] Abtahizadeh, E., van Oijen, J., de Goey, P., "Numerical study of MILD combustion with entrainment of burned gas into oxidizer and/or fuel streams", Combust. Flame, 159: 2155-2165 (2012) 
[8] Sabia, P., de Joannon, M., Picarelli, A., Ragucci, R., "Methane auto-iognition delay times and oxidation regimes in MILD combustion at atmospheric pressure", Combust. Flame, 160: 47-55 (2013)

[9] Sabia, P., de Joannon, M., Picarelli, A., Chinnici, A., Ragucci, R., "Modeling negative temperature coefficient region in methane oxidation", Fuel, 91: 238-245 (2012)

[10] Oberlack, M., Arlitt, R., Peters, N., "On stochastic Damkohler number variations in a homogeneous flow reactor", Combust. Theor. Model., 4: 495-509 (2000).

[11] Mastorakos, E., Taylor, A.M.K.P., Whitelaw, J. H., "Extinction of turbulent counterflow flames with reactants diluted by hot products", Combust. Flame, 102: 101-114 (1995).

[12] Bray, K. N. C., Champion, M., Libby, P. A., "Extinction of premixed flames in turbulent counterflowing streams with unequal enthalpies", Combust. Flame, 107: 53-64 (1996).

[13] Kee, R. J., Rupley, F. M., Miller, J. A., Coltrin, M. E., Grcar, J. F., Meeks, E., Moffat, H. K., Lutz, A. E., Dixon-Lewis, G., Smooke, M. D., Warnatz, J., Evans, G. H., Larson, R. S., Mitchell, R. E., Petzold, L. R., Reynolds, W. C., Caracotsios, M., Stewart, W. E., Glarborg, P., Wang, C., Adigun, O., CHEMKIN Collection, Release 3.6, Reaction Design, Inc., San Diego, CA (2000)

[14] Smith, G. P., Golden, D. M., Frenklach, M., Moriarty, N. W., Eiteneer, B., Goldenberg, M., Bowman, C. T., Hanson, R. K., Song, S., Gardiner, W. C. Jr., Lissianski, V. V., Qin Z., GRI 3.0, <http://www.me.berkeley.edu/gri_mech/>

[15] Law, C. K., Combustion Physics, Cambridge University Press, 2006.

[16] Minamoto, Y., Dunstan, T. D., Swaminathan, N., Cant, R. S., "DNS of EGR-type turbulent flames in MILD combustion", Proc. Combust. Inst., 34: 3231-3238 (2013)

[17] Robinson, C., Smith, D. B., "The auto-ignition temperature of methane", J. Hazard. Mater., 8: 199-203 (1984).

[18] Caron, M., Goethals, M., De Smedt, G., Berghmans, J., Vliegen, S., Van't Oost, E., van den Aarssen, A., "Pressure dependence of the auto-ignition temperature of methane/air mixtures", J. Hazard. Mater., 65: 223-244 (1999). 